\title{
Identificación de riesgos por desastres naturales en las cuencas hidrográficas Matasnillo (Panamá) y Goascorán (Honduras), y su relación con el cambio climático utilizando Tecnologías de Información Geográfica
}

Eduardo L. Moreno Segura, Claudia N. Mondragón Rivera, José D. Cáceres Coello, Antonio B. Carias Arias

\section{Resumen}

Honduras y Panamá están caracterizados por precipitaciones intensas y de larga duración, tormentas, fuertes descargas eléctricas, inundaciones, incendios de masas vegetales, trompas marinas, tsunamis, episodios de la presencia de El Niño, La Niña y de derrames de sustancias peligrosas. El presente trabajo se desarrolla en el marco del Programa Universitario para la Gestión Integral del Riesgo de Desastres y Adaptación al Cambio Climático en Centroamérica(PRIDCA) coordinado por el Consejo Superior Universitario Centroamericano y financiado por la Agencia Suiza para el Desarrollo y la Cooperación -COSUDE-, y donde la Universidad Nacional Autónoma de Honduras y la Universidad Marítima Internacional de Panamá a través de un proyecto interuniversitario han decidido desde una perspectiva de investigación científica y tecnológica, establecer estrategias y acciones para la reducción de riesgos de desastres naturales en las cuencas hidrográficas de los Ríos Matasnillo, Golfo de Panamá, y la cuenca del Río Goascorán, Golfo de Fonseca, Honduras; y la relación del cambio climático que además comprenda un eje transversal de involucramiento y apropiación del proceso por parte de los actores claves en las zonas de estudio, mediante el desarrollo de una metodología que facilite la identificación, el análisis y la clasificación de riesgos producto de la identificación de amenazas y vulnerabilidades en el área de las cuencas seleccionadas a través de la aplicación de tecnologías de Información 
Geográfica, elaborando además propuestas y recomendaciones para minimizar riesgos identificados como altos en base a profundidad de la amenaza.

Palabras Clave: Tecnologías de Información Geográfica, riesgos, desastres, amenazas, cambio climático, cuenca.

\section{Abstract}

Honduras and Panama are characterized by intenseand long-last ingrainfall, storms, severe electrical shock, floods, wildfires, marinetubes,tsunamis, episodes of the presence of El Niño, La Niña and spills of hazardous substances. On the other hand, Global climate change model sindicate that both countries are subject to severe changes, such as increased temperature, drought,more intense precipitation,more frequent stormsand rising average sea level. This work is carried out under the University Program for Comprehensive Disaster Risk Managementand Adaptation to Climate Changein Central America(PRIDCA)coordinated by the Central American University Council(CSUCA) and funded by the Swiss Agency for Development and Cooperation (COSUDE)- and where the National Autonomous University of Honduras and the International Maritime University of Panama, through an interuniversity project decided, from the perspective of scientificand technological research, to develop strategies and actions to reduce risks of natural disasters in watersheds Rivers Matasnillo, Gulf of Panama,and the Goascorán River basin, Gulf of Fonseca, Honduras; and the relationship of climate change further comprising a transverseaxisof involvement and owner ship of theprocess by the key players in the study areas, through the development of a methodology that enables the identification, analysis and classificationofrisk product identifying threatsand vulnerabilities inthe basins selectedthrough theapplicationof GIS technologies, as well as developing proposals and recommendations tominimize risks identifie dashigh based on depth threat.

Keywords: Geographic Information Technologies, risks, disasters, threats, climate change, basin.

Eduardo L. Moreno Segura, (eddmorse7@gmail.com,) Claudia N. Mondragón Rivera, (cn.mondragonrivera@gmail.com), José D. Cáceres Coello, (josedavid.caceres@gmail. com,) Antonio B. Carias Arias, (,antoniocarias@gmail.com). Departamento de Ciencia y Tecnologías de la Información Geográfica,Facultad de Ciencias Espaciales, Universidad Nacional Autónoma de Honduras. 


\section{INTRODUCCIÓN}

El propósito del proyecto parte del enfoque a la gestión del riesgo, este tiene una amplitud de orden político y técnico social dirigida a la reducción del riesgo. Estas acciones encaminan a las municipalidades a la generación de legislaciones normativas para la preparación ante catástrofes.

El enfoque busca la reflexión de la sociedad local para maximizar la colaboración a nivel de gobiernos locales y que lleguen a tomar decisiones correctas para reducir el riesgo y su posterior efecto al ocurrir el desastre actuando así de mejor manera ante una inundación, incendio forestal, deslizamientos, terremotos y sequía.

Este proyecto pretende brindar la línea base y herramientas básicas prácticas para hacer que la reducción del riesgo sea un éxito a nivel de gobiernos locales y ha contribuido mediante el desarrollo de una metodología que posibilite el análisis y la clasificación de riesgos producto de la identificación de posibles amenazas y nivel de vulnerabilidad en las áreas de estudio (cuenca hidrográfica marino costera del Río Goascorán, Golfo de Fonseca Honduras, y cuenca hidrográfica marino costera del Río Matasnillo, Golfo de Panamá) todo esto mediante el uso de la tecnologías de Información Geográfica, elaborando además propuestas, recomendaciones y transferencias de capacidades a los beneficiarios del proyecto para minimizar riesgos identificados como altos.

\section{METODOLOGÍA}

La metodología empleada consistió en un recorrido por todos los municipios de influencia del proyecto para establecer una fase de socialización sobre los estudios a realizar, los beneficios que los resultados del proyecto traerían a la comunidad al contar con un documento y herramientas que les permita conocer e identificar los riesgos climáticos a los cuales se enfrentan y utilizar las estrategias para mitigar y adaptarse al impacto del cambio climático; finalmente se desarrollaron talleres técnicos con los involucrados de cada municipalidad y actores claves en ambos países, los cuales brindaron información pertinente a la dinámica de afectación en el territorio, al mismo tiempo se elaboraron los principales mapas de amenazas que sirvieron como base fundamental en la construcción de la base de datos digital de riesgos en el territorio. 
El proyecto de investigación fue planteado para realizarse en 6 fases metodológicas, mismas a desarrollarse a lo largo de 12 meses.

Fase I Preparación Preliminar, esta fase consiste en la preparación del equipo de trabajo y primer acercamiento formal a la zona de estudio.

Fase II Diagnóstico Situacional, en esta fase de desarrollaran los diagnósticos situacionales necesarios para establecer cuál es la situación actual en las cuencas hidrográficas, identificar la problemática y las oportunidades existentes, con el fin de delimitar las zonas específicas para aplicar los estudios posteriores.

Fase III Identificación de Amenazas, en esta fase metodológica se procederá a la identificación de las principales amenazas de la cuenca tomando como base las zonas específicas a estudiar dentro de las cuencas hidrográficas.

Fase IV Identificación de Vulnerabilidades, una vez identificadas las amenazas, en esta fase se procederá a identificar las vulnerabilidades territoriales existentes en las cuencas hidrográficas tomando como base las actividades humanas e infraestructuras tales como: ciudades, vías de comunicación, infraestructura de salud y educación, infraestructura económica, actividades económicas, áreas protegidas, densidad poblacional.

Fase V Identificación de Riesgos, teniendo como base las amenazas y las vulnerabilidades se procederá a la identificación de riesgos, que se resumen en la siguiente ecuación: Riesgos= amenazas * vulnerabilidad.

Fase VI Propuestas y Recomendaciones, en esta fase se propondrán recomendaciones y propuestas para minimizar aquellos riesgos identificados como medios y altos en las cuencas hidrográficas, con el fin de no contar únicamente con un inventario de riesgos si no de proponer una solución integral a los riesgos identificados.

Existen además 3 fases metodológicas transversales de vital importancia para el desarrollo del proyecto:

- Fase transversal de cooperación interuniversitaria.

- Fase transversal de participación ciudadana.

- Fase transversal de transferencia de capacidades locales. Ver Figura 1. 
Proceso metodológico de la investigación.

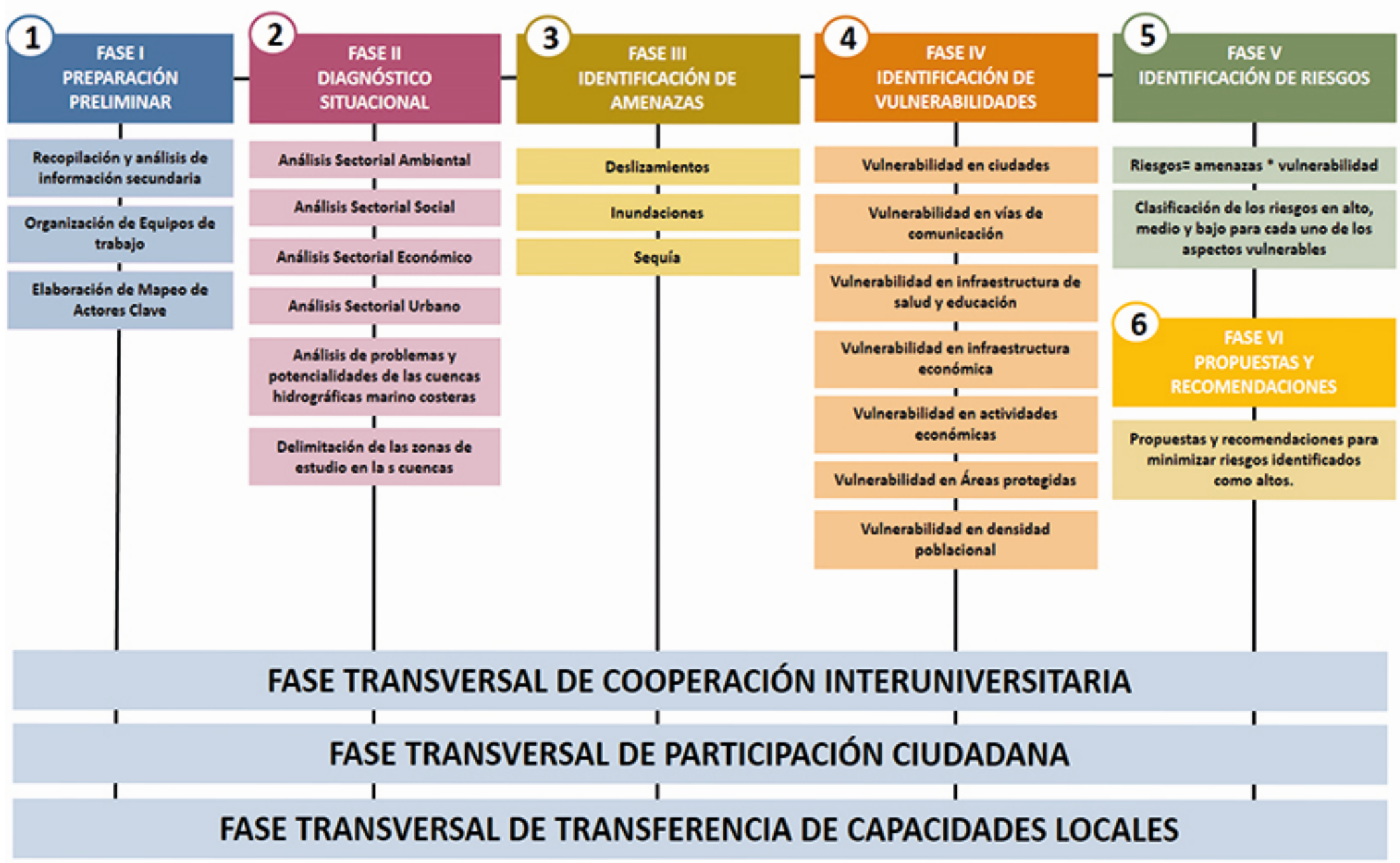

Figura 1.Proceso Metodológico de la Investigación.

Variables y su Operacionalización:

Considerando que el propósito de investigación del proyecto es Desarrollar una metodología que posibilite la identificación, el análisis y la clasificación de riesgos producto de la identificación de amenazas y vulnerabilidades en el área de la cuenca hidrográfica mediante el uso de la tecnologías de Información Geográfica, Las variables a investigar fueron: Inundación, Sismos, Deslizamientos, Sequía y Propensión a Incendios. 


\section{En la Tabla 1 Operacionalización y operacionalizan las variables se definen estos detalles.}

Tabla 1. Operacionalización y conceptualización de variables.

\begin{tabular}{|c|c|c|}
\hline VARIABLE & $\begin{array}{l}\text { DEFINICION } \\
\text { CONCEPTUAL }\end{array}$ & DEFINICION OPERACIONAL \\
\hline Inundacion & $\begin{array}{l}\text { La amenaza por inundación } \\
\text { se da en función de la } \\
\text { probabilidad de ocurrencia } \\
\text { del evento y su intensidad, } \\
\text { ésta se puede definir en } \\
\text { función de la profundidad y } \\
\text { velocidad del agua, asi } \\
\text { como en la duración de las } \\
\text { inundaciones. }\end{array}$ & $\begin{array}{l}\text { La identificación de las amenazas a inundación de los } \\
\text { municipios que intervienen en la Cuenca del Rio } \\
\text { Goascoran se desarrolla mediante el abordaje de dos } \\
\text { diferentes procedimientos: Talleres comunitarios y } \\
\text { Análisis de planicies de inundación mediante } \\
\text { procedimientos geomorfológicos. }\end{array}$ \\
\hline Sismos & $\begin{array}{l}\text { Nivel probable de } \\
\text { movimiento de la terra } \\
\text { asociado a la recurrencia a } \\
\text { un terremoto. }\end{array}$ & $\begin{array}{l}\text { Para identificar el riesgo asociado a los terremotos } \\
\text { recurrentes se calculó un mapa global al peligro de } \\
\text { sismos producido por el Global } \\
\text { SeismicHazardAssesmentProgram (GSHAP). }\end{array}$ \\
\hline Deslizamientos & $\begin{array}{l}\text { Es un tipo de corrimiento o } \\
\text { movimiento de masa de } \\
\text { tierra, provocado por la } \\
\text { inestabilidad de un talud. }\end{array}$ & $\begin{array}{l}\text { Se aplicó mediante la combinación de varios factores y } \\
\text { parámetros, los cuales se obtienen de la observación y } \\
\text { medición de indicadores morfodinámicos y su } \\
\text { distribución espacio-temporal. La combinación de los } \\
\text { factores y parámetros se realiza considerando que los } \\
\text { deslizamientos ocurren cuando en una ladera, } \\
\text { compuesta por una Itología determinada, con cierto } \\
\text { grado de humedad y con cierta pendiente, se alcanza } \\
\text { un grado de susceptibilidad (elementos pasivos). }\end{array}$ \\
\hline Sequia & $\begin{array}{l}\text { Involucra algún tipo de } \\
\text { disminución en algún } \\
\text { periodo de tiempo y este se } \\
\text { analiza únicamente con } \\
\text { precipitación. }\end{array}$ & $\begin{array}{l}\text { El ISSP (Indice de Sequia de Palmer) es un } \\
\text { procedimiento que se calibra para regiones } \\
\text { homogéneas, es un indicador meteorológico y } \\
\text { responde a condiciones climáticas que han sido } \\
\text { anormalmente secas o anormalmente húmedas, } \\
\text { basándose en la oferta y demanda del balance hidrico. }\end{array}$ \\
\hline $\begin{array}{l}\text { Propension a } \\
\text { Incendios }\end{array}$ & $\begin{array}{l}\text { Se define como el fuego } \\
\text { que se extiende sin control } \\
\text { en terrenos forestal } \\
\text { afectando a combustibles } \\
\text { vegetales }\end{array}$ & $\begin{array}{l}\text { Se consideraron tres aspectos fundamentales para ser } \\
\text { incluidos al modelo de propensión de incendios, la cual } \\
\text { pretende establecer el nivel de propensión (o ser sujeto } \\
\text { de presencia de incendios) en base a factores de } \\
\text { acceso y las diferentes categorias de cobertura del } \\
\text { suelo, considerando sus factores inherentes de la } \\
\text { relativa faciidad de ser afectado por incendios de } \\
\text { acuerdo a la naturaleza de los materiales } \\
\text { predominantes u la facilidad de los mismos de ignición. }\end{array}$ \\
\hline
\end{tabular}




\section{RESULTADOS}

\section{Universo, Población y Muestra:}

Esta investigación se constituye como una investigación de campo aplicada, abordada mediante un proceso formal. La naturaleza de los datos a utilizar es de metodología cuantitativa (áreas específicas afectadas en cada municipio) y de metodología cualitativa (encuestas de opinión y de percepción).

Esta es una investigación orientada a decisiones ya que no se centra en hacer aportes teóricos, más bien su objetivo primordial es buscar soluciones a los problemas encontrados.

El tamaño de la población beneficiada es 84,809 hombres y 95,400 mujeres distribuidos en 5 municipios en Honduras y 3 Corregimientos en Panamá, de estos la muestra para obtener la información cualitativa fue de aproximadamente 200 mediante el desarrollo de talleres participativos tanto en Honduras como en Panamá.

\section{PROCEDIMIENTOS Y TÉCNICAS (estimación de las áreas específicas afectadas en cada municipio)}

Para la estimación de las áreas específicas afectadas en cada municipio se modelaron, mediante la aplicación de tecnologías de la información geográfica, diferentes amenazas de origen natural en el área de estudio. Las amenazas son las posibles tragedias a las que se está expuesto y que nos pueden causar un daño con cierta intensidad en el futuro, basado en un antecedente que es repetido en ciclos durante varios años y tienen alta probabilidad de materializarse inesperadamente.

En este proyecto de investigación se analizaron las amenazas de incendios forestales, sequia meteorológica, deslizamientos, sismos e inundaciones mediante un proceso técnico científico y otro mediante talleres comunitarios los cuales validaban el resultado preliminar en escritorio con los modelamientos de las diferentes amenazas.

El resultado de todo este proceso de investigación fue la elaboración de mapas claves para la planificación territorial e insumo importante para la evaluación del riesgo futuro. 
Para la estimación de índice de sequía es importante definir que existen tres tipos de sequía:

- Meteorológica: La que involucra algún tipo de disminución en algún periodo de tiempo y este se analiza únicamente con precipitación.

- Hidrológica: esta se refiere a la reducción de los recursos acuáticos en algún periodo de tiempo.

- Agrícola: que es el impacto de las dos anteriores en las actividades humanas.

La identificación de la intensidad de la sequía es importante para la prevención en la planificación agrícola de la zona de un país, es por ello que partiendo de un trabajo de depuración y consolidación de la base de datos de las estaciones climatológicas de la zona sur se procedió a calcular el índice de Sequia de Palmer. No se obtuvieron datos climáticos de Panamá.

EI ISSP (Índice de Sequia de Palmer) es un procedimiento que se calibra para regiones homogéneas, es un indicador meteorológico y responde a condiciones climáticas que han sido anormalmente secas o anormalmente húmedas, basándose en la oferta y demanda del balance hídrico (Ver Figura 2).

En el balance hidrológico se estima la evapotranspiración potencial (ETP), la recarga $(\mathrm{R})$, el escurrimiento (Ro), pérdida de humedad de suelo (L) y los potenciales valores. Se determinaron los siguientes coeficientes: a o Coeficiente de Evapotranspiración (ET / PE), ß o Coeficiente de Recarga (R / PR), y o Coeficiente de Escurrimiento (RO / PRO), $\delta$ o Coeficiente de Pérdidas (L / PL). Utilizando estos coeficientes se determinó la cantidad de humedad climática o normal para cada mes de acuerdo a esta ecuación:

$$
\widehat{P}=E \hat{t}+\widehat{R}+R \hat{o}+\hat{L}
$$

La diferencia entre la precipitación actual y la precipitación necesaria para mantener el promedio de humedad climática o normal Palmer la define como apartamiento de humedad (d).

$$
\mathrm{d}=\mathrm{p}-\hat{\mathrm{p}}
$$

Durante los períodos húmedos estos apartamientos son positivos y durante los secos son negativos. Además obtuvo un índice de anomalías de humedad ( $\mathrm{z}$ ) 
multiplicando los valores $d$ por una constante $k$ determinada para cada lugar y para cada período $(z=d . k)$.

Se estimó como la relación demanda-suministro de humedad y la representó según:

$$
\mathrm{k}=\overline{\mathrm{ETP}}+\overline{\mathrm{R}} / \overline{\mathrm{P}}+\overline{\mathrm{L}}
$$

Los rangos de periodos húmedos y secos según Palmer se detallan en la Tabla 2.

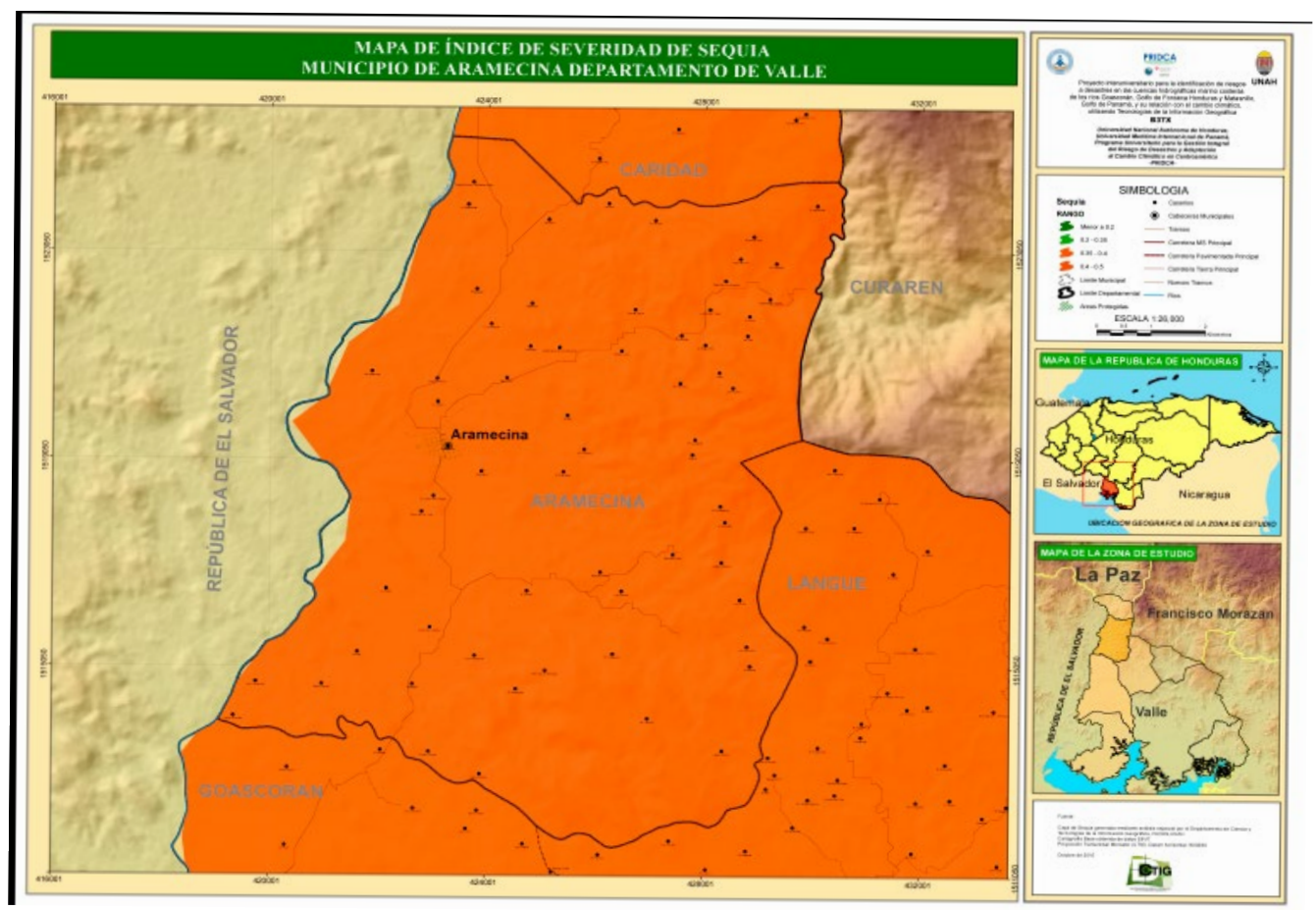

Figura 2. Mapa de Índice de Severidad de Sequía. Municipio de Aramecina, Departamento de Valle. 
Tabla 2. Rangos de periodos húmedos y secos según Palmer (1965)

\begin{tabular}{|l|l|}
\hline \multicolumn{1}{|c|}{$\mathrm{X}$} & \multicolumn{1}{c|}{ Clases } \\
\hline$>4,0$ & Humedad extrema \\
\hline 3,0 a 3,9 & Humedad severa \\
\hline 2,0 a 2,9 & Humedad moderada \\
\hline 1,0 a 1,9 & Ligeramente húmedo \\
\hline 0,5 a 0,9 & Humedad incipiente \\
\hline$-0,4$ a 0,4 & Normal \\
\hline$-0,5$ a $-0,9$ & Sequía incipiente \\
\hline$-1,0$ a $-1,9$ & Sequía reducida \\
\hline$-2,0$ a $-2,9$ & Sequía moderada \\
\hline$-3,0$ a $-3,9$ & Sequía severa \\
\hline$<-4,0$ & Sequía extrema \\
\hline
\end{tabular}

Las inundaciones provocan enormes daños entre los que se encuentran pérdidas en las vidas humanas, en la economía, en el sector agrícola y productivo, así como al sector infraestructura como en vivienda y transporte.

La amenaza por inundación se da en función de la probabilidad de ocurrencia del evento y su intensidad y duración, ésta se puede definir en función de la profundidad y velocidad del agua, así como en la duración de las inundaciones.

Honduras cuenta con un mapa nacional de susceptibilidad a inundaciones, elaborado por el Instituto Hondureño de Ciencias de la Tierra de la Universidad Nacional Autónoma de Honduras. Este análisis fue realizado tomando en cuenta factores como la geomorfología y la topografía; definiendo a través de la aplicación de Sistemas de Información Geográfica (SIG), las zonas susceptibles a inundaciones.

La identificación de las amenazas a inundación de los municipios que intervienen en la Cuenca del Rio Goascoran y los corregimientos de la cuenca del Río Matasnillo (Ver Figuras 3 y 4.), se desarrolla mediante el abordaje de dos diferentes procedimientos:Talleres comunitarios.

- Análisis de planicies de inundación mediante procedimientos geomorfológicos.

Para el desarrollo del primer Ítem, se desarrollaron una serie importantes de talleres en los cuales representantes de diferentes comunidades, quienes se 
sirvieron de los mapas provistos, sobre los cuales delimitaron las zonas afectadas por los fenómenos naturales de mayor incidencia en el municipio. Este principio participativo sirvió para el desarrollo de los mapas de memoria comunitaria de afectación de los principales fenómenos naturales que de manera recurrente han afectado a los municipios de la zona de estudio.

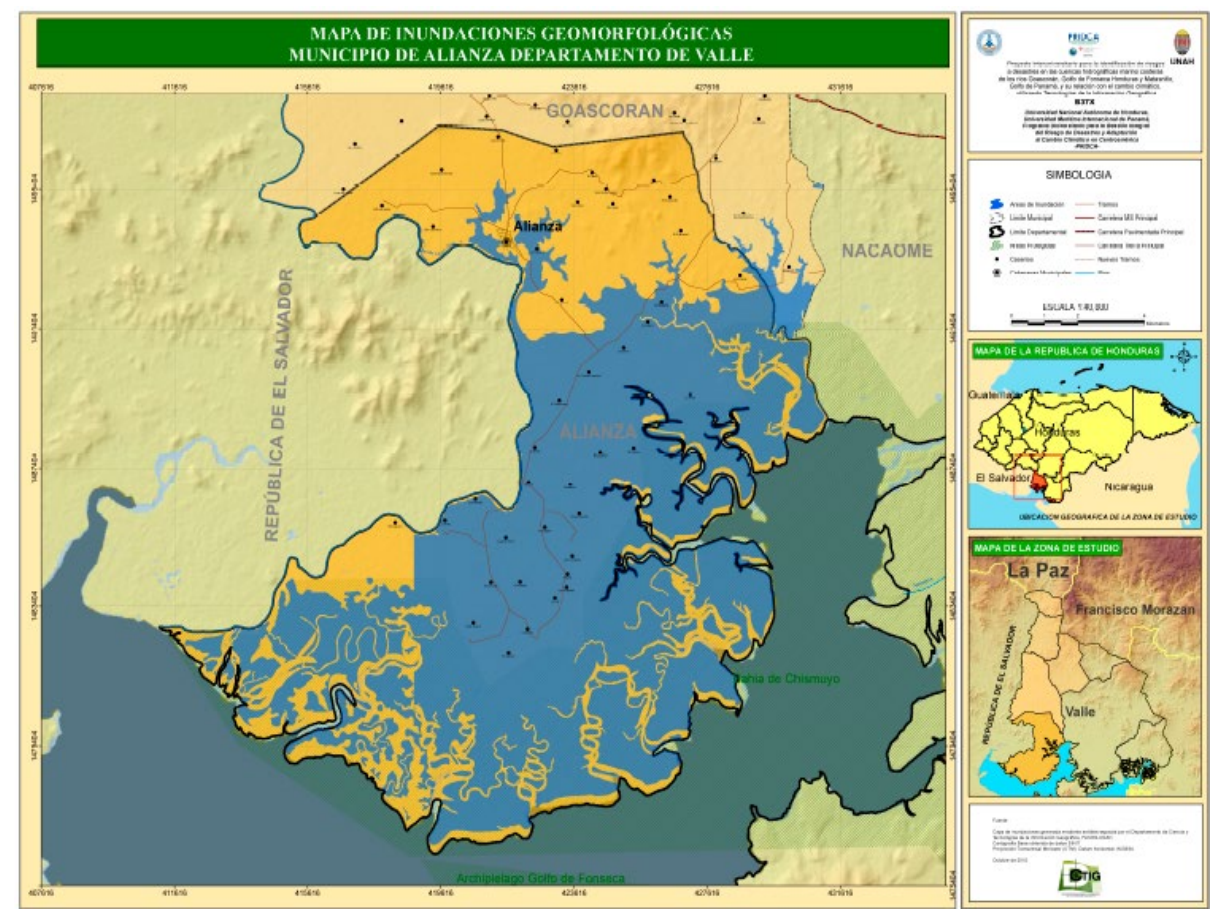

Figura 3. Mapa de Inundaciones Geomorfológicas. Municipio de Alianza, Departamento de Valle.

En Panamá se aplicó la misma metodología para construir el mapa de Amenaza por Mareas y Marejadas. 


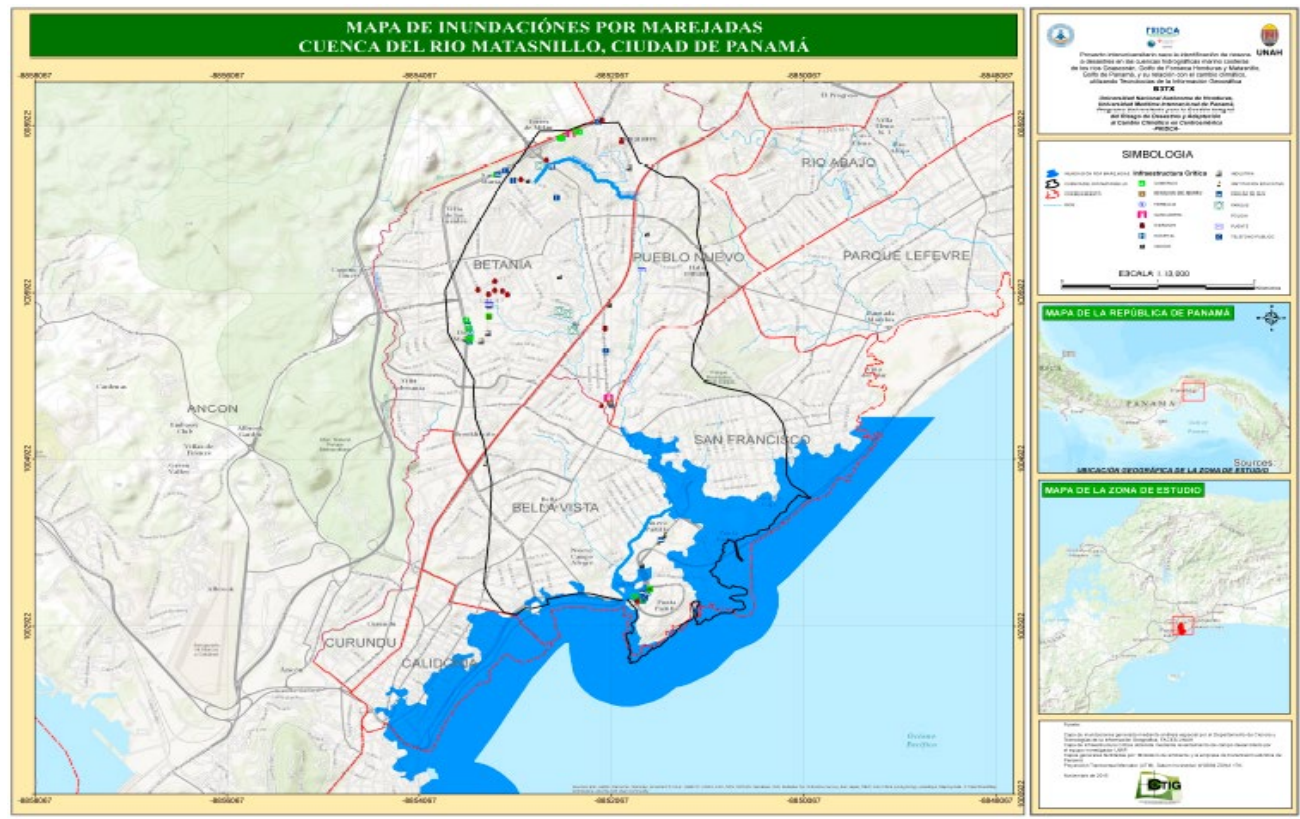

Figura 4. Mapa de Inundaciones por Marejadas. Cuenca del Río Matasnillo, Ciudad de Panamà.

Para la generación del Mapa de Propensión a Incendios Forestales (ver Figuras 5 y 6.), se consideraron tres aspectos fundamentales para ser incluidos al modelo de propensión de incendios, la cual pretende establecer el nivel de propensión (o ser sujeto de presencia de incendios) en base a factores de acceso y las diferentes categorías de cobertura del suelo, considerando sus factores inherentes de la relativa facilidad de ser afectado por incendios de acuerdo a la naturaleza de los materiales predominantes u la facilidad de los mismos de ignición. Bajo este concepto, una categoría de matorrales presenta un nivel de susceptibilidad 0 propensión a incendios Alta, la cual puede llegar a ser mayor si se encuentra en una zona de accesibilidad alta, lo que significa que dicha categoría de cobertura de suelo estaría con una propensión de incendio Muy Alta, por el mayor nivel de exposición a tráfico de personas, lo que aumenta las probabilidades de incendio. Bajo un mismo concepto de espera que un Bosque Latifoliado con un nivel de acceso Difícil, su nivel de Propensión sea Bajo, comparativamente. 
De manera general, para el desarrollo de este mapa se consideraron los siguientes factores:

- Cobertura del Suelo

$\begin{array}{ll}\text { - } & \text { Red Vial } \\ \text { - } & \text { Pendientes } \\ & \text { Puntos de Calor MODIS }\end{array}$

Para efecto de relacionar las categorías de Cobertura del Suelo con los niveles de accesibilidad, de manera general se consideró la Tabla 3 matriz de evaluación en la cual se establecen tres categorías de propensión: Alta (3), Media (2) y Baja (1): 
Tabla 3. Matriz de Evaluación

\begin{tabular}{|c|c|c|c|}
\hline Capa de Entrada & Ponderación & Definición & Escala \\
\hline \multirow{15}{*}{$\begin{array}{l}\text { Mapa Forestal y } \\
\text { Cobertura del } \\
\text { Suelo } 2014\end{array}$} & \multirow[t]{15}{*}{$50 \%$} & Bosque Latifoliado Seco & 2 \\
\hline & & Bosque Mixto & 2 \\
\hline & & Pino Denso & 2 \\
\hline & & Pino Ralo & 2 \\
\hline & & Mangle Alto & 1 \\
\hline & & Mangle Bajo & 1 \\
\hline & & Vegetación Secundaria Seca & 3 \\
\hline & & Agricultura Tecnificada & 3 \\
\hline & & Pastos y/o Cultivos & 3 \\
\hline & & Otros cuerpos de Agua & 1 \\
\hline & & Zonas Urbanizadas discontinuas & 3 \\
\hline & & Suelos Desnudos Continentales & 3 \\
\hline & & Áreas Húmedas Continentales & 1 \\
\hline & & Camaroneras y Salineras & 1 \\
\hline & & Arboles Dispersos & 1 \\
\hline \multirow[t]{6}{*}{ Red Vial } & \multirow[t]{6}{*}{$15 \%$} & Afirmado sólido, dos o más vías & 3 \\
\hline & & $\begin{array}{l}\text { Revestimiento suelto } 0 \text { ligero, } \\
\text { dos o más vías }\end{array}$ & 3 \\
\hline & & $\begin{array}{l}\text { Revestimiento suelto o ligero, } \\
\text { una vía }\end{array}$ & 2 \\
\hline & & $\begin{array}{l}\text { Revestimiento suelto, transitable } \\
\text { solo en verano }\end{array}$ & 2 \\
\hline & & Sendero o vereda & 1 \\
\hline & & Vereda de rodada & 1 \\
\hline \multirow[t]{3}{*}{ Pendientes } & \multirow[t]{3}{*}{$15 \%$} & $0-10 \%$ & 3 \\
\hline & & $10-30 \%$ & 2 \\
\hline & & $>30 \%$ & 1 \\
\hline $\begin{array}{l}\text { Densidad Puntos } \\
\text { de Calor MODIS }\end{array}$ & $20 \%$ & & \\
\hline TOTAL & $100 \%$ & & \\
\hline
\end{tabular}

Bajo este concepto, las categorías tales como Matorrales, Pastizales y Sabanas, Agricultura Migratoria (entre otras) poseen una propensiónAlta, la cual al combinarse con un nivel de accesibilidad alta, incrementa sustancialmente las probabilidades de convertirse en zonas de Propensión Alta a incendios. 
Estos modelos pretenden definir el nivel de exposición incendios basados en aspectos de acceso y categorías de cobertura del suelo, la cual se estima basado en el Mapa Forestal y de Cobertura del Suelo 2014 elaborado por el Instituto de Conservación y Desarrollo Forestal, Áreas Protegidas y Vida Silvestre para los municipios evaluados.. En el caso de Panamá se utilizó la capa de Uso de la Tierra y Red Vial proporcionadas por la UMIP.

Finalmente se obtiene el mapa de propensión de incendios, en el cual se puede apreciar que aquellas categorías de cobertura del suelo que están próximas a las zonas de influencia de las principales vías de acceso, exhiben un mayor nivel de propensión a incendios, mientras que categorías de uso de suelo correspondientes a Bosques con niveles de acceso difícil, presentan un nivel de propensión Bajo.

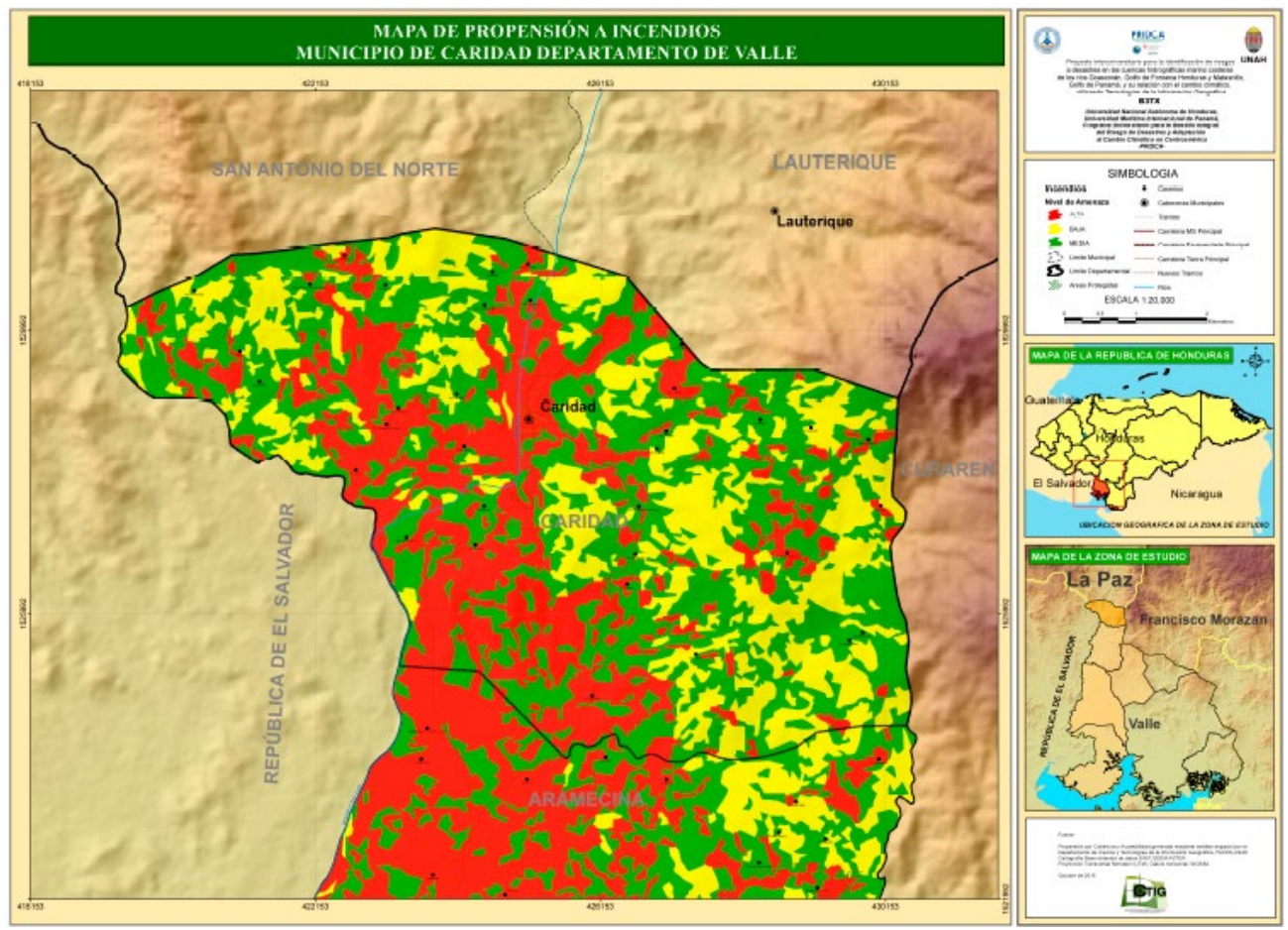

Figura 5. Mapa de Propensión a Incendios. Municipio de Caridad, Departamento de Valle. 


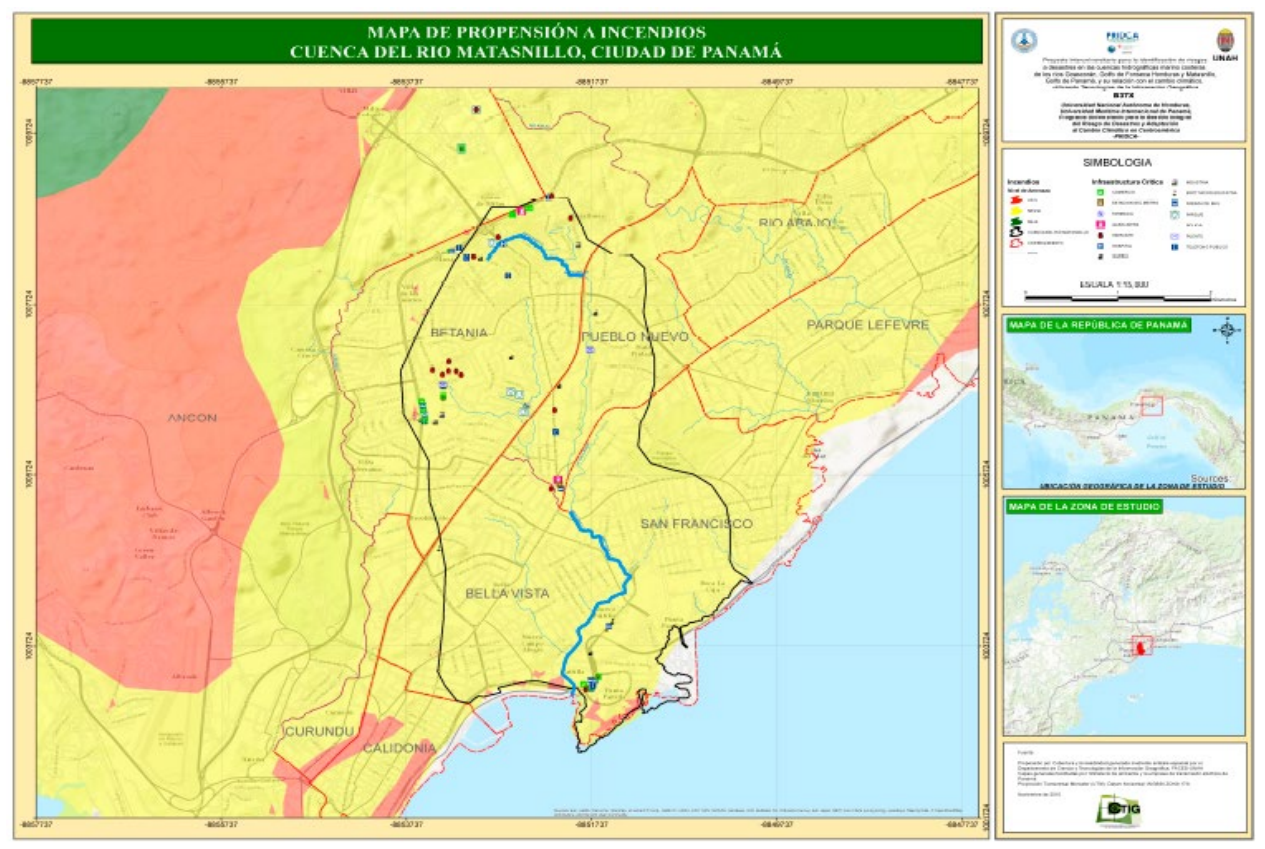

Figura 6. Mapa de Propensión a Incendios. Cuenca del Río Matasnillo, Ciudad de Panamá.

El peligro a sismos es definido como el nivel probable de movimiento de la tierra asociado a la recurrencia a un terremoto (ver Figuras 7 y 8.).

Con el sentido de mitigar el riesgo asociado a los terremotos recurrentes se calculó un mapa global al peligro de sismos producido por el Global Seismic Hazard Assesment Program (GSHAP).

La estrategia del GSHAP es minimizar las pérdidas de vidas humanas, perdida de viviendas y problemas económicos y sociales debido a la presencia del sismo. 


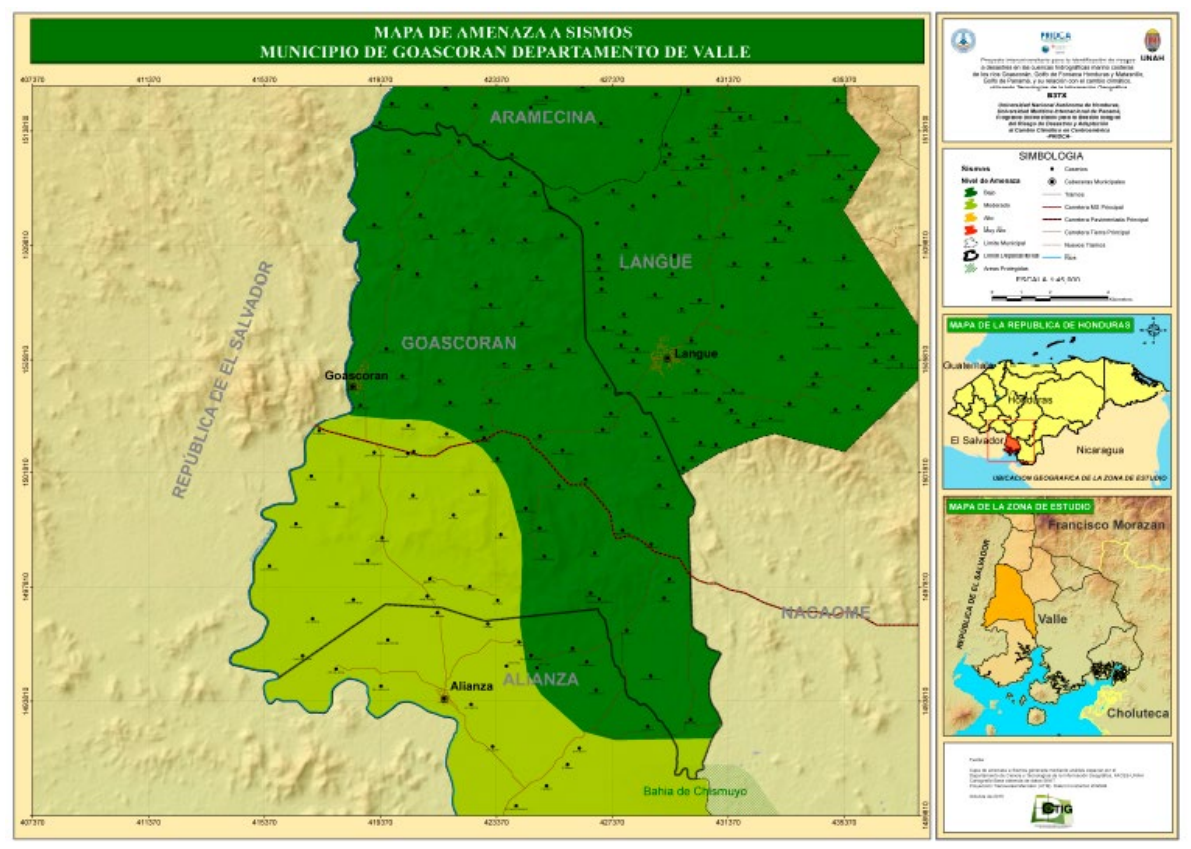

Figura 7. Mapa de Amenaza a Sismos. Municipio de Goascorán, Departamento de Valle.

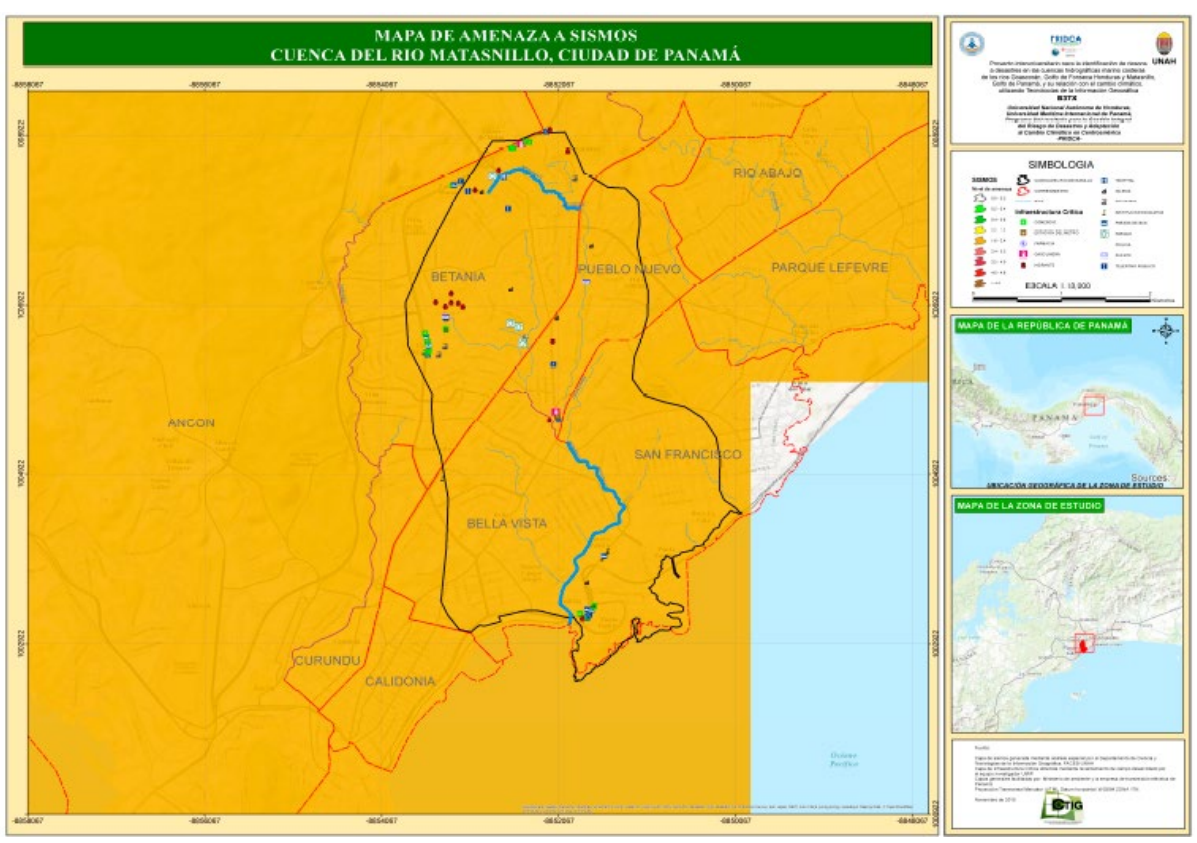

Figura 8. Mapa de Amenaza a Sismos. Cuenca del Río Matasnillo, Ciudad de Panamá 
Para la modelación de Amenaza a Deslizamientos se utilizó el método Mora \& Vahrson (ver Figura 9.).

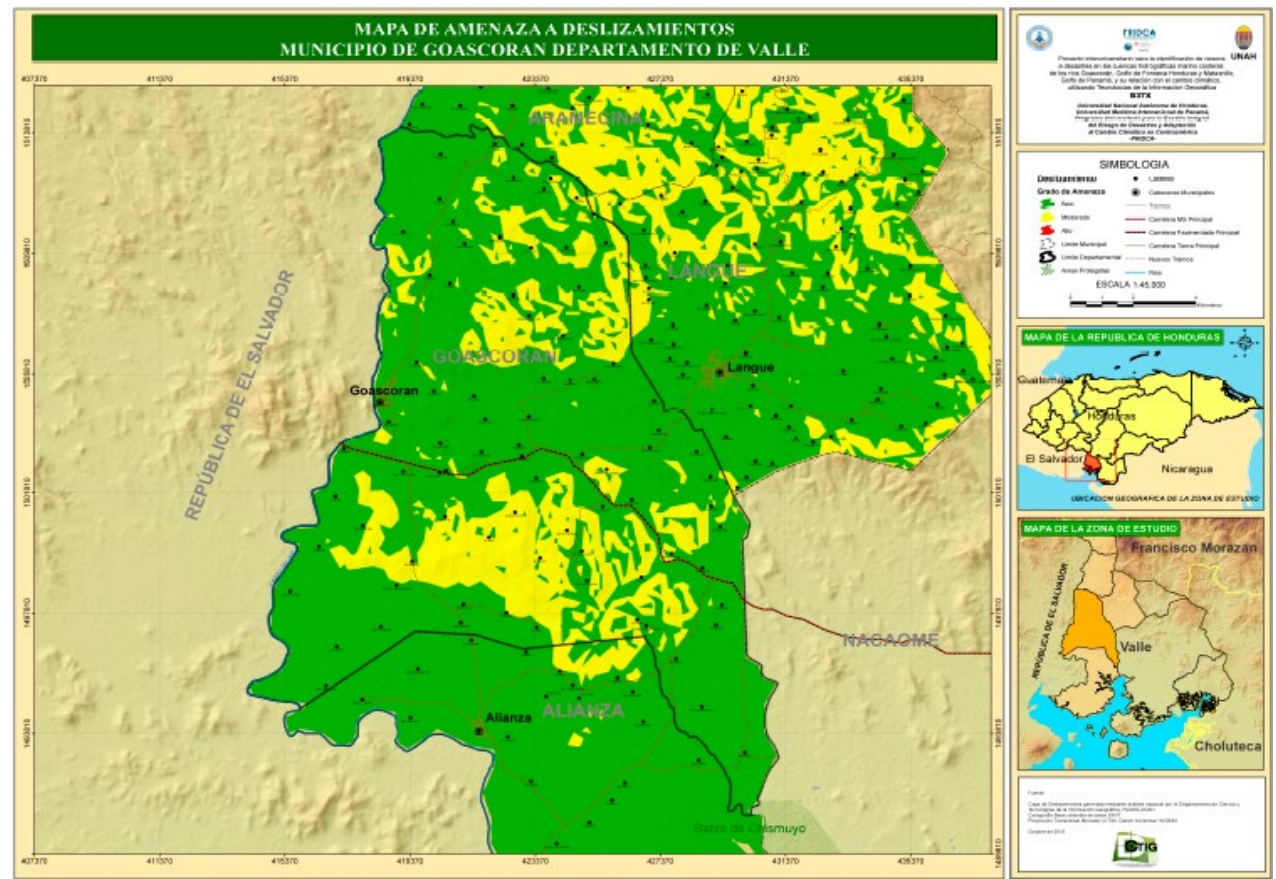

Figura 9. Mapa de Amenaza a Deslizamientos. Municipio de Goascorán, Departamento de Valle.

Este método es elaborado en Costa Rica, por Mora y Wilhelm-Guenther Vahrson, constituye una importante referencia para clasificar la amenaza por deslizamientos, o lo que se denomina "movimientos de remoción en masa", y ha sido ampliamente utilizado, principalmente en Centroamérica. Sin embargo, éste sólo va a constituir una referencia para los modelos de susceptibilidad y amenaza, en la escala de valores que proponen, ya que ofrece una clasificación que va del 1 al 5 , utilizando calificativos que van desde el nivel de susceptibilidad muy baja, baja, moderada, alta y muy alta (Segura, Badilla, \& Obando, 2011).

Se aplica mediante la combinación de varios factores y parámetros, los cuales se obtienen de la observación y medición de indicadores morfodinámicos y su distribución espacio-temporal. La combinación de los factores y parámetros se realiza considerando que los deslizamientos ocurren cuando en una ladera, compuesta por una litología determinada, con cierto grado de humedad y con cierta 
pendiente, se alcanza un grado de susceptibilidad (elementos pasivos). Bajo estas condiciones, los factores externos y dinámicos, como son la sismicidad y las lluvias intensas (elementos activos), actúan como factores de disparo que perturban el equilibrio, la mayoría de las veces precario, que se mantiene en la ladera.

Es así como se considera que el grado de amenaza al deslizamiento es el producto de los elementos pasivos y de la acción de los factores de disparo, de acuerdo con la siguiente fórmula:

$\begin{array}{ll}\text { - } & \text { Factores intrínsecos (Susceptibilidad) } \\ \text { - } & \text { Relieve relativo (Sr) } \\ \text { - } & \text { Litología (SI) } \\ \text { - } & \text { Humedad del Suelo (Sh) } \\ \text { - } & \text { Factores externos (Disparo) } \\ \text { - } & \text { Precipidad Sísmica (Ts) }\end{array}$

Nivel de Amenaza relativa

$$
\begin{array}{ll}
\text { - } & H=S U S C * D I S P \\
\text { - } & H=\left(S L{ }^{*} S H * S R\right) *(T s+T p)
\end{array}
$$

Donde:
- SL: valor del parámetro de susceptibilidad litológica,
- SH: valor del parámetro de humedad del terreno,
- SR: valor del parámetro del relieve
- Ts: valor del parámetro de disparo sismológico y
- $\quad T p:$ valor del parámetro de disparo por lluvia




\section{Factores}

\section{Factor topográfico $(\mathrm{Sr})$}

Para el área de estudio se trabajará con un modelo de elevación digital de 90 metros de píxel, del cual se derivará la información topográfica, de esta forma para el método Mora-Vahrson se pueden utilizar tanto la pendiente del terreno como el relieve relativo.

\section{Pendiente}

La pendiente identifica la máxima inclinación de una localización sobre una superficie. Sobre el modelo de elevación del terreno la pendiente se calcula como la máxima tasa de cambio de la elevación sobre cada celda y sus ocho vecinos más próximos.

En SIG el cálculo de la pendiente corresponde a la primera derivada aplicada a un modelo numérico digital del terreno (MNT), así a partir de la superficie o MNT de entrada en m.s.n.m se calcula una archivo de salida que contiene la pendiente para cada celda; los valores más bajos corresponde a terrenos más planos, los valores más altos a terrenos con pendientes más fuertes.

Cuando el ángulo de la pendiente es 45 grados, la altura de escalada vertical es igual al desplazamiento horizontal. Expresado como porcentaje, la pendiente de este ángulo es $100 \%$. Mientras que cuando la pendiente se acerca a la vertical (90 grados), la pendiente del porcentaje tiende a infinito.

En la Tabla 4 se muestra el calificativo de las pendientes que recomienda la metodología.

Tabla 4. Calificativo del factor de pendientes

\begin{tabular}{|l|l|l|}
\hline \multicolumn{1}{|c|}{ Pendiente (\%) } & \multicolumn{1}{|c|}{ Valoración del parámetro $(\mathrm{P})$} & \multicolumn{1}{c|}{ Grado de susceptibilidad } \\
\hline $0-7,5$ & 0 & Muy baja \\
\hline $7,5-17,5$ & 1 & Baja \\
\hline $17,5-30$ & 2 & Moderada \\
\hline $30-50$ & 3 & Mediana \\
\hline $50-80$ & 4 & Alta \\
\hline Más de 80 & 5 & Muy alta \\
\hline
\end{tabular}


El procedimiento en Sistemas de Información Geográfica (SIG) es muy simple, a partir del modelo numérico del terreno se genera el mapa de pendientes en porcentaje y de aquí se reclasifica para obtener el factor de pendientes. La información que provee el mapa de pendientes es bastante valiosa, sobre todo en lo concerniente a la evaluación de la inestabilidad de las laderas, dependiendo de su constitución geológica y otros factores.

El valor de las pendientes surge de un subproceso del Modelo de Elevación Digital, aplicando SIG, para obtener el factor de pendientes que se detalla en la Tabla 5.

Tabla 5. Valor del factor de la pendiente (Sr)

\begin{tabular}{|l|l|}
\hline \multicolumn{1}{|c|}{$\begin{array}{c}\text { Valor del factor de la pendiente } \\
(\mathrm{Sr})\end{array}$} & ha \\
\hline $\mathbf{0}$ & 36988 \\
\hline $\mathbf{1}$ & 14481 \\
\hline $\mathbf{2}$ & 10969 \\
\hline $\mathbf{3}$ & 5549 \\
\hline $\mathbf{4}$ & 627 \\
\hline $\mathbf{5}$ & 1 \\
\hline
\end{tabular}

Factor Geología - litología (SI)

El factor litológico SI es probablemente el más relevante y a la vez el más difícil de valorar. Idealmente se debiera usar información geotécnica detallada. Cuando esta información no está disponible, como en el caso de la Región de la cuenca baja del rio Goascoran, se puede usar una descripción basada en la geología. La descripción y las comparaciones deben ser desarrolladas con un buen juicio geotécnico.

Sin embargo, para la optimización del método se toman en cuenta los siguientes parámetros para cuantificar y clasificar cada tipo de roca. Composición mineralógica, capacidad de retención de humedad, espesor y grado de meteorización, nivel de fracturación, buzamientos, posición y variabilidad de los niveles freáticos, entre otros.

Para el caso de la Región del río Goascorán se realizó la clasificación a partir del mapa de Geología realizado a nivel nacional y se detalló por un geólogo con calicatas en la zona de estudio. 
Unidades estratigráficas

- Qt. - Terrazas del Cuaternario

- Ky.- Calizas Cretácicas del Grupo Yojoa

- Kj.- Calizas Cretácicas Jaitique del Grupo Valle de Ángeles

- Ke.- Calizas Cretácicas Esquías del Grupo Valle de Ángeles

- Kg.- Calizas Cretácicas Guare del Grupo Valle de Ángeles

- Kva. - Areniscas rojas, arcillas rojas o lutitas, limolitas y conglomerados de cuarzo del Cretácico Valle de Ángeles.

- Jhg. - Areniscas y lutitas grises con trazas de carbón del jurásico Grupo Honduras.

- Tpm. - Rocas volcánicas del Terciario Padre Miguel

- Tm. - Rocas volcánicas del Terciario Matagalpa (andesitas y basaltos)

- Trm . - Rocas volcánicas del Terciario Tipo Río Manto (andesitas porfídicas grises)

- Kti. - Rocas intrusivas del Cretácico

- Pzm. - Rocas metamórficas del Paleozoico (todos los esquistos; gneises; pizarras y cuarcitas)

- Qd. - Depósitos lacustres como las rocas volcánicas de la Formación Gracias (Tg)

- Qt. - Terrazas del Cuaternario

- Tcg. - Rocas volcánicas del Terciario Cerro Grande

- Tg. - Rocas volcánicas de la Formación Gracias.

- Qal. - Cuaternario aluvión; Qc. - Cuaternario coluvión. 


\section{Factor humedad (Sh)}

Para este mapa se utilizó los mapas del balance hídricos. Mora y Vahrson , simplifican aún más los balances hídricos, requiriendo para ello únicamente la información de la precipitación promedio mensual. Categorizando los promedios mensuales de acuerdo la Tabla 6.

Tabla 6. Clasificación de los promedios mensuales

\begin{tabular}{|l|l|}
\hline \multicolumn{1}{|c|}{ Precipitación promedio (mm) } & Valor asignado \\
\hline Menor de 125 & 0 \\
\hline $125-250$ & 1 \\
\hline Mayor de 250 & 2 \\
\hline
\end{tabular}

El factor de humedad del suelo es importante ya que involucra con las altas temperaturas a la meteorización lo que genera una porosidad en la roca generando una mayor propensión al deslizamiento, al final se suman los valores mensuales dando el valor acumulado al año.

\section{Actividad sísmica (Ts)}

El peligro a sismos es definido como el nivel probable de movimiento de la tierra asociado a la recurrencia a un terremoto.

Con el sentido de mitigar el riesgo asociado a los terremotos recurrentes se calculó un mapa global al peligro de sismos producido por el Global Seismic Hazard Assesment Program (GSHAP).

La estrategia del GSHAP es minimizar las pérdidas de vidas humanas, perdida de viviendas y problemas económicos y sociales debido a la presencia del sismo.

La zona de intervención del proyecto entra dentro de la categoría 2.4 - 4.0 lo que significa según la escala definida que está en una zona de Alta amenaza ya que los valores de 0 - 0.8 es amenaza baja, $0.8-2.4$ es amenaza moderada, $2.4-4.0$ es de amenaza alta y de 4.0 - 5 es de amenaza muy alta.

Es importante tenerbien definido para los tomadores de decisiones la implementación de planes con estrategias para mitigar los eventos sísmicos en esta zona ya que están en una escala alarmante y de mucha precaución. 
Factor precipitación (Tp)

Para determinar la intensidad de las lluvias se utilizan datos de estaciones meteorológicas de valores de lluvia máximos en un periodo de retorno de 50 o 100 años con estas estadísticas se elabora un mapa mediante interpolación para tener la distribución superficial del dato estadístico con la precipitación promedio anual. La Tabla 7 detalla la clasificación de precipitación.

Tabla 7. Calificación precipitación

\begin{tabular}{|l|l|l|}
\hline $\begin{array}{c}\text { Precipitación máxima, para } \mathrm{n}>\mathbf{1 0}, \mathrm{Tr}=100 \\
\text { años }\end{array}$ & \multicolumn{1}{|c|}{ Calificación } & Factor Tp \\
\hline$<100 \mathrm{~mm}$ & Muy baja & 1 \\
\hline $100-200$ & Baja & 2 \\
\hline $200-300$ & Media & 3 \\
\hline $300-400$ & Alta & 4 \\
\hline$>400$ & Muy alta & 5 \\
\hline
\end{tabular}

Mapa Final de Amenaza a Deslizamiento

El factor de susceptibilidad de amenaza a deslizamiento parte de la formula antes descrita en la cual mediante algebra de mapas se establece el mapa final. Este mapa permitirá determinar las áreas de mayor amenaza de deslizamientos, siguiendo la clasificación de grados de amenaza de deslizamiento de Mora \& Varhson. La Tabla 8 especifica la clasificación del nivel de amenaza a deslizamiento.

Tabla 8. Clasificación del nivel de amenaza

\begin{tabular}{|l|l|l|}
\hline $\begin{array}{c}\text { Potencial de amenaza (pesos } \\
\text { susceptibilidad *disparo) }\end{array}$ & Clave & Grado de amenaza \\
\hline $0-6$ & I & Muy Bajo \\
\hline $6-32$ & II & Bajo \\
\hline $32-162$ & III & Moderado \\
\hline $162-512$ & IV & Mediano \\
\hline $512-1250$ & $\mathrm{~V}$ & Alto \\
\hline Mayor que 1250 & $\mathrm{VI}$ & Muy Alto \\
\hline
\end{tabular}




\section{CONCLUSIONES Y RECOMENDACIONES}

- En los municipios de la Cueca Baja del Río Goascorán en Honduras, después del análisis cualitativo y cuantitativo se determinó que existe para este territorio 5 amenazas: a inundaciones, deslizamientos, sequía, incendios y sismos. En el caso de Panamá se identificaron 3 amenazas: mareas y marejadas, propensión a incendios y sismos.

- La amenaza de sequía para los 5 municipios en Honduras se encuentra dentro de un rango medio-alto. La sequía, al igual que las inundaciones, provocan grandes daños a la economía y a las familias que ha afectado. Sólo en la sequía que se presentó en el 2001, se estima que el país perdió el $23 \%$ de la producción de maíz, el $35 \%$ de la de frijol y $60 \%$ de sorgo. Sólo en las regiones centro, sur y occidental se perdieron alrededor de 3 millones de quintales de granos básicos, provocando un desabastecimiento significativo en la dieta de la población.

- En la amenaza de inundación en Honduras, Alianza tiene un mayor puntaje en propensión a inundaciones debido a su cercanía al mar, en contraste con Langue el cual al no tener ni un afluente cercano no presenta ni un porcentaje grave a esta amenaza. En el caso de Panamá solamente dos de los cuatro corregimientos analizados presentaron susceptibilidad a esta amenaza.

- En la amenaza de propensión a incendios, se determina que el $43 \%$ del territorio comprendido por los 5 municipios de la Cuenca Baja del Río Goascorán tiene una propensión alta a incendios forestales, y el 30\% una propensión media. Bajo este concepto, las categorías tales como Matorrales, Pastizales y Sabanas, Agricultura Migratoria (entre otras) poseen una propensión Alta, la cual al combinarse con un nivel de accesibilidad alta, incrementa sustancialmente las probabilidades de convertirse en zonas de Propensión Alta a incendios. En el caso de Panamá apenas un $0.21 \%$ presenta propensión alta a incendios.

- En la amenaza a sismos La zona de intervención del proyecto entra dentro de la categoría 2.4 - 4.0 lo que significa según la escala definida que está en una zona de Alta amenaza ya que los valores de 0 - 0.8 es amenaza baja, 0.8 2.4 es amenaza moderada, $2.4-4.0$ es de amenaza alta y de $4.0-5$ es de amenaza muy alta. El Panamá la categoría identificada corresponde a la escala de 1.6 - 2.4 categorizada como amenaza moderada. 
- La amenaza a deslizamientos se da sobre todo en los territorios altos de los municipios de Aramecina, Caridad, Goascorán y Langue, siendo este alrededor del $30 \%$ del territorio de la Cuenca Baja.

- Para la solución de los problemas, amenazas y riesgos encontrados para los 5 municipios de la cuenca baja del Rio Goascorán se desarrollarón 5 propuestas a implementarse en los municipios, estas van desde la implementación de una campaña en contra de los incendios forestales, un plan de lucha contra la desertificación, construcción de cosechas de agua, Sistema de gestión de cuenca baja (alerta temprana y monitoreo) ante desastres naturales. Las anteriores desarrolladas a nivel de ficha.

- Es importante el acompañamiento que han dado al proceso las autoridades locales de los municipios (en Honduras) y corregimientos (en Panamá) involucrados, así como las instituciones gubernamentales y de cooperación internacional que tienen presencia en las cuencas de intervención.Este es fundamental para diseñar estrategias locales de intervención y disminuir los efectos de estos fenómenos.

- Este acompañamiento por parte de los actores clave de las cuencas asegura un respaldo institucional hacia la investigación y una validez a los datos que se han recolectado durante todo el proceso.

- Asegura además que el instrumento que se ha generado, será de utilidad para las instituciones encargadas de la toma de decisiones sobre la temática de gestión de riesgos en los municipios y zonas estudiadas.

- De suma importancia para la trascendencia y continuidad del proyecto ha sido la etapa transversal de transferencia de capacidades que se ha venido llevando a cabo con los técnicos municipales y la participación activa de estudiantes, así como la apropiación y conciencia de la temática de gestión de riesgos y adaptación al cambio climático por parte de los pobladores de las zonas estudiadas.

- Con este proyecto queda la lección aprendida de prever muchos de los topes administrativos que antes se desconocían (y que incluso algunos fueron creándose en el camino) y que se encuentran en cada institución, garantizando así la ejecución en tiempo y forma de las actividades que dependen del presupuesto. 


\section{AGRADECIMIENTOS}

Este artículo fue hecho como producto del Proyecto interuniversitario para la identificación de riesgos por desastres naturales en las cuencas hidrográficas marino costeras de los ríos Matasnillo, Golfo de Panamá, y la río Goascorán, Golfo de Fonseca Honduras, y su relación con el cambio climático utilizando Tecnologías de la Información Geográfica" (con número de identificador B37X) ejecutado por la Universidad Nacional Autónoma de Honduras (UNAH) y La Universidad Marítima Internacional de Panamá (UMIP) y auspiciado por el "Programa Universitario para la Reducción de Riesgo de Desastres y Adaptación al Cambio Climático en Centro América -PRIDCA, el Consejo Superior Universitario Centroamericano (CSUCA) y financiada con fondos de la Cooperación Suiza (COSUDE).

\section{BIBLIOGRAFIA}

- Masciangioli Pánfilo (2011). Amenazas Naturales en el Área Marino Costera de Venezuela. Primera Jornada sobre seguridad de los Próceres. Puerto La Cruz, Estado Anzoategui, Segura, G., Badilla, E., \& Obando, L. (2011). SUSCEPTIBILIDAD AL DESLIZAMIENTO EN EL CORREDOR SIQUIRRESTURRIALBA. Revista Geológica de América Central, 45, 101-121.

- FAO (2011). Estrategia para la Pesca, La Acuicultura y el Cambio Climático. Marco de Trabajo y objetivos 2011-2016. Departamento de Pesca y Acuicultura de la FAO.

- BID (2002). Amenaza, Vulnerabilidad, Riesgo, Desastre, Mltigación, Prevención, Programa de Información de Indicadores de Gestión de Riesgo. BID, CEPAL, IDEA.

- OPS (1999). Manual de Monitoreo de la Ejecución de Proyectos. Oficina de Relaciones Externas, Organización Mundial de la Salud.

- Gómez Natalia (2009), Análisis de Riesgos de desastres y Vulnerabilidad en la República Dominicana. OXFAM, Comisión Europea.

- INE (2001), Censo Población y Vivienda INE 2001, Honduras.

- Brito Lorenzo (2008). Introducción a los Conceptos de Manejo Integrado de Zonas Costera y Cuencas Hidrográficas-. México. 
- Torres, J. (2005). Reflexiones del V curso internacional sobre el manejo de SIG para La mitigación de los riesgos de desastres. [Artículo en línea]. Disponible: http://geofocus.rediris.es/2005/Informe7_2005.pdf. [Consulta: 2006, diciembre 19].

- García, J., Monnar, O., Zapata, J., Arango, E., y López, P. (2006). Sistema de Información Geográfica para el manejo y evaluación del riesgo sísmico en la ciudad de Santiago de Cuba. [Artículo en línea]. Disponible: http://www.espejos. unesco.org.uy/simplac2002/ponencias/geom\%E1tica\%202 002/GEO50.doc. [Consulta: 2006, diciembre 17]. 